\title{
ORIOINAL PAPER
}

\section{Using Structural Equation Modelling (SEM) to predict use of Voluntary Counselling and Testing (VCT) services among adults in Zambia}

\author{
Mutale $\mathrm{W}^{1,2}$, Michelo $\mathrm{C}^{1,2}$, Sam D.L ${ }^{3}$ \\ ${ }^{\prime}$ Department of Community Medicine, School of Medicine, University of Zambia \\ ${ }^{2}$ Centre for International Health, University of Bergen, Norway \\ ${ }^{3}$ Department of Psychosocial Science, University of Bergen, Norway
}

\section{ABSTRACT}

Background: Voluntary Counselling and Testing (VCT) has become a cornerstone intervention in the fight against HIV. Current evidence suggests that it is cost-effective in changing behaviour and preventing HIV infection. A number of studies have demonstrated some of the factors which are associated with both intention and actual use of VCT services. However, studies which show how various factors combine to influence intention and subsequently the use of VCT services are generally lacking.

We employed Structural Equation Modelling (SEM) to examine the relationship between factors involved in use of VCT services.

Method: The AMOS program version 6 was used for our data analysis and data from a population based survey in Zambia (2003) was used to fit the model.

Results: The proposed model fitted well for the population. Fitness indices fell within accepted ranges; GFI $=0.998, \quad$ AGFI $=0.994$ and RMSEA $=0.025$. The model fitted well across age group (young/older participants), residence (rural/urban) and sex (male and female participants). All standardised regression weights were significant at $95 \%$ confidence level except for regression weight between self-rated health and willingness. Psychological distress was positively associated

\footnotetext{
*Corresponding author:

Mutale Wilbroad,

Department of Community Medicine, School of Medicine,

University of Zambia,

Box 50110, Lusaka, Zambia,

Email:wmutale@yahoo.com.
}

with health use, depression, self- rated risk and willingness (intention), but negatively associated with self-rated health. Willingness (intention) was positively associated with participation in VCT.

Conclusion: SEM was successfully used to explore how various determinants interact to influence the use of VCT services and most of our theoretical assumptions were supported by empirical findings. Self-rated health was a key explanatory parameter for psychological distress and the intention to use VCT services.

\section{INTRODUCTION}

Voluntary Counselling and Testing (VCT) has become a critical factor in the fight against HIV. The benefits of VCT cut across HIV status as it takes care of both the infected and uninfected individuals ${ }^{1,3}$. It is also an entry point for treatment and prevention of mother to child transmission of HIV ${ }^{4,6}$. It was found to be effective in changing behaviour and studies have shown that it is cost-effective and compares favourably with other interventions ${ }^{7,9}$.

Recently, World Health Organisation (WHO) and other partners have embarked on a programme to scale up delivery of antiretroviral drugs (ARVs) to those living in low-income countries. The obvious obstacle is the low number of people who know their HIV status. It is therefore crucial that the scale up is matched with the increased uptake of VCT services if these efforts are to be successful ${ }^{2,3}$. Many barriers have prevented maximisation of the benefits of VCT. Studies have demonstrated some of the factors

Key words

Voluntary counselling and testing (VCT), Structural equation modelling (SEM), AMOS program Introduction 
which are associated with both intention and actual use of VCT services. These factors include high risk behaviour, poor self-rated health, home delivery of test results, gender and residence, ${ }^{10,18}$ among others.

However, studies which show how various factors combine to influence intention and subsequent use of VCT services are generally lacking. This analysis can help to identify exact points which require modification in order to improve the uptake of VCT services.

Structure Equation Modelling (SEM) is one method which can be used to create a model with various components predicting behaviour or intention. It has been used in many research areas, but has scarcely been applied to VCT.

We employed SEM using AMOS programme version 6 to examine a VCT model using data from a population survey in Zambia to fit the model.

A two factor confirmatory analysis was initially modelled with intention and psychological distress as the two components. This fitted well and a final model was created with "coming to test for HIV" as the behaviour to predict.

\section{Theoretical basis of the VCT model}

This model stems from previous studies which showed that "self-rated health, increased risk, and depression" (Mutale et al, unpublished) were associated with the use of VCT services. Some of these factors have been shown to independently affect the intention to use VCT as well ${ }^{12}$. In this study, a model which links the various factors involved in determining the utilisation of VCT was therefore proposed.

The following description is a theoretical explanation of how the various components are presumed to influence each other:

Poor self-rated health may lead to increased use of health services, increasing contact with health workers who may raise awareness about HIV and VCT thereby influencing the intention ${ }^{19,20}$. At the same time, poor self-rated health may influence the intention (willingness) directly, as poor health in the era of HIV may trigger worries about the possibility of being infected ${ }^{12}$. In addition to being related to poor self-rated health, increased use of health services may result in psychological distress because of concerns about the possibility of being infected with HIV, especially if one has high risk behaviour ${ }^{21}$, ${ }^{22}$. Depression is another factor thought to influence the intention through psychological distress. Depression may either be a direct result of worries or other illnesses that comes with HIV infection. It may also be the effect of the virus on the patients' mental state $^{23,26}$.

Psychological distress is the main mediator between other factors and the intention to come for VCT (willingness). All components act directly or indirectly via intention (willingness) in order to influence the behaviour of coming for VCT. This is because VCT is voluntary and clients must willingly make a decision to take the HIV test without compulsion ${ }^{27}$. Hence, the variance contributed by willingness is expected to be substantial because all of the other components are represented. Socioeconomic and demographic factors are thought to modify the components in the model as background variables.

\section{METHODOLOGY}

\section{Study areas and population}

The model was tested on data collected in a population-based, cross-sectional survey conducted in Zambia, 2003. The survey employed stratified random-cluster sampling of selected communities in an urban area of Lusaka and a rural area of Kapiri Mposhi. The survey used structured questionnaires and face-to-face interviews to collect information from both men and women aged 15-59 on matters pertaining to their socio-demographic, health and sexual behaviour. Details of participation rates are reported elsewhere ${ }^{28}$. The initial number of participants was 5035, of which 4302 were retained for analysis after excluding those with missing values.

\section{VCT study design}

At the end of the survey interviewed respondents were informed that the saliva-based HIV test was strictly for research purposes and would be handled anonymously. Furthermore, they were informed about the general strategy regarding VCT in Zambia and how services could be arranged for them as part of the survey. Those who expressed interest in being tested (willingness or readiness) were then followed 
up by counsellors who were part of the survey. The counsellors provided pretest counselling and collected blood samples for testing. Post test counselling was also provided by the counsellors. All of this was done at home. All services were offered free of charge, but no particular strategy was instituted in terms of long-term follow-up services to HIV-infected individuals other than providing information about existing support and care opportunities. The counsellors recorded all outcome information $^{12,28}$.

\section{Measures}

Came for VCT; was the actual number of people who came for VCT and were precounselled and tested for HIV. (Recoded as YES or NO ${ }^{1,2}$.

Willingness (intention); was evaluated by a single question:" Would you like us to arrange for you to be tested for HIV?"Answers were YES or NO ${ }^{1,2}$.

Self-rated health; was based on single item: "How would you say your health is at the moment?" Scored 1 to 4, (excellent, good, fair, and poor).

Self-rated risk; was assessed by 2 question; " "In your situation do you think that your are at risk of getting (catching) HIV?" Scored 1 to 4 (No risk, moderate risk, high, and very high risk). " ${ }^{2}$ How worried are you about actually being infected with HIV". Score 1 to 4 (never, seldom, sometimes and, always worried. (Croabach's alpha was 0.53 ).

Depression; was based on sum score on four dichotomous variables from the Beck Depression Inventory (BDI) ${ }^{29}$; Do you; (a) sleep badly, (b) find it difficult to enjoy life activities, (c) ever thought of ending your life and (d) feel tired always. Answers were Yes or No $(1,0)$. Croabach's alpha was 0.50 .

Health service use score; was based on three dummy variables; in the last one year; (i) have you been admitted to the hospital (ii) visited the clinic (iii) visited the hospital, Croabach's alpha was 0.46 .

We used Root mean square error of approximation (RMSEA), adjusted goodness-of-fit index (AGFI) and Goodness-of-fit index (GFI) to assess how well the data fitted the proposed model. Chi-square is not recommended when the sample size is large $e^{30}$.

\section{RESULTS}

Demographic characteristics of participants:

The majority of respondents were males (58.4\%) and urban residents (61.3\%). Most were from middle economic class (63.6\%) and $45 \%$ were aged 15-24 years of age. Single and married were equally represented at $46 \%$ and the rest were either widowed or divorced. $32 \%$ of participants expressed willingness (intention) and $26 \%$ of the participants came for VCT. The majority of participants had low health and depression scores. Most respondents $(56 \%)$ rated their health as good and about one-third rated their risk as "very low" and "high" respectively.

Mean scores for self-rated health was 3.08 $(\mathrm{SD}=0.71)$, while the mean for self-rated risk was $2.47(\mathrm{SD}=1.28)$. Depression and health use scores had lower means and these results are shown in Table 1.

\section{Table 1: Descriptive statistics}

\begin{tabular}{llll}
\hline & N & Mean & $\begin{array}{c}\text { Std. } \\
\text { Deviation }\end{array}$ \\
\hline Self rated health & 4302 & 3.08 & 0.71 \\
Self rated risk & 4302 & 2.47 & 1.28 \\
Depression score & 4302 & 0.46 & 0.81 \\
Came for HIV testing & 4302 & 1.74 & 0.44 \\
Willing to come for VCT & 4302 & 1.68 & 0.47 \\
Health services use index & 4302 & 0.22 & 0.47 \\
\hline
\end{tabular}

\section{Correlation between variables}

VCT (behaviour) was positively correlated with willingness (intention), $\mathrm{p}<0.001$ ). However, coming for VCT and willingness were negatively correlated with self-rated risk, depression, health use, and selfrated health. All of the correlations were significant at $95 \%$ confidence level. (See Table 2).

\section{Structural equation modelling}

The proposed model fitted well for the population. The fitness indices fell within accepted ranges; Chi square was $0.001, \mathrm{GFI}=0.998, \mathrm{AGFI}=0.994$ and RMSEA $=0.025$. Group comparison demonstrated that the model fitted well across age group (young/older participants), residence (rural/urban) and sex (male/ female).

GFI and AGFI values greater than 0.95 and RMSEA values less than 0.05 are indicative of good fitness ${ }^{30}$. 
All standardised regression weights were significant at $95 \%$ confidence level except for the regression weight between self-rated health and willingness.

Associations: Psychological distress was positively associated with health use, depression, self-rated risk and willingness (intention), but was negatively associated with self-rated health. Willingness (intention) was positively associated with self-rated health and coming for HIV testing. Most indirect effects to coming for HIV testing were mediated through psychological distress.

\section{DISCUSSION}

In our study, structural equation modelling was used to explore how various determinants interact to influence the use of VCT services. The model fitted very well, first for the whole study population and also across age group, sex and residence. Moreover, most of our theoretical assumptions where supported by empirical findings. As predicted, psychological distress exerted a direct positive effect on the intention (willingness) to come for VCT. The direct positive effect of the level of depression, self-rated risk and health use on psychological distress was also predicted. This is plausible because the intention to come for testing is a reflection of the psychological evaluation of the cost and benefits of taking a test ${ }^{20}$. In addition, the likelihood of a positive test given the risk behaviour, poor health (as indicated by frequent use of health services) and depression all influence the mental state as measured by psychological distress. These factors acting together can influence the intention to come for VCT. This view was supported by our empirical findings in the model.

The finding that those with high risk tend to come for VCT more than those with low risk perception was observed in other studies and seems to be supported by our model ${ }^{12,20}$. Individuals with high risk may be more likely to use VCT services in order to know their HIV status and allay fears of being infected. It is noteworthy that in addition to risk, depression has also been reported in HIV patients, though its aetiology has not yet been elucidated. Factors associated with depression in HIV patients include physical limitations, stigma, sexual orientation and religion in homosexuals ${ }^{24,26}$. Our model supported our assumption that depression is positively associated with psychological distress. The theory of learned helplessness proposed by Seligman (1970) and reformulated by (Gilbert, 1984), is an important theoretical consideration which attempts to explain depression in helpless people ${ }^{31}$. The central idea in the learned helplessness theory is that all animals (including humans) are able to learn that reinforcers are uncontrollable. According to this theory, depression will occur if the individual is aware of uncontrollable factors in their environment, views the situation as unchangeable and blame themselves for their helplessness (internal attribution). In the study of the HIV epidemic, the theory of learned helplessness has been used before in African Americans and seemed to predict depression ${ }^{32}$. The depression reported in our study could therefore be related to this theory especially with stigma and limited availability of a cure for the HIV infection in Africa.

Furthermore, increased use of health services was positively related to psychological distress as predicted. People who use health services frequent are usually in poor physical and possibly mental health which will be reflected in their level of psychological distress.

Contrary to our prediction, self-rated health was negatively associated with psychological distress. One possible explanation is that those with extremely poor health have given up hope and are indifferent about the distress related to their illness or they have come to accept their situation. However, self-rated health was positively and directly associated with the intention to come for VCT as has been reported in other studies and was confirmed in our model ${ }^{12}$.

Finding that intention (willingness) was positively and directly associated with coming for VCT, was expected because those individuals who have voluntarily accepted the VCT are ready to come for testing ${ }^{27,33}$. The model also revealed that most indirect effects of coming for VCT were mediated through psychological distress. This was expected as most components of the model loaded on psychological distress.

The finding that those with increased psychological distress resulting from high risk behaviour, 
depression or frequent illness (as indicated by poor health and increased frequency of using health services) appear to benefit more from VCT services suggests that VCT may be missing an important section of the population who think they are at less risk, in good health and unworried about HIV infection.

HIV prevalence was almost equal (16 vs. 17\%) in this same study population for the groups viewing themselves as high and low risk. Misinterpretation of risk may reflect ignorance, denial or other social constructs. Previous studies observed that people may perceive themselves to be at low risk even though objective risk factors indicate otherwise $\mathrm{e}^{21,34}$. For example, condom use in the last year was low for those who thought that they were at low risk in this study population. Thus, it is clear that individuals rating themselves as low risk are actually at high risk and in need of VCT services. Hence, efforts must be directed towards increasing risk perception in the population. This can lead to increased concern about the possibility of infection, intention and subsequent coming for HIV testing,

Another intervention of "opt out" seems to be supported by our model. Increased use of health services was found to influence intention via psychological distress.

In "opt out" method VCT is given as part of the routine clinical tests to all patients. This method can also be very useful in improving uptake of VCT especially if it is done as part of risk awareness campaign during counselling sessions ${ }^{35,36}$.

Our study has some limitations. It was based on VCT offered at home, so its applicability to other settings is limited. However, as most indicators used were similar to those reported in hospital or satellite (stand-alone) VCT centres, we feel that our findings can also apply to these settings. SEM usually employs scales in assessing the loading of various components in the model. Our study used dichotomous variables in the assessment of intention and the behaviour of coming to test, therefore this must be taken into consideration when interpreting the model. Our cross-sectional design also diminishes our capacity to draw causal inferences from the results. A longitudinal design is better suited for this. The reliability scores appear to be low for the scales employed in this study. However, they do not necessarily affect the results of the model as demonstrated by excellent fitting indices ${ }^{37}$. Despite the above limiting factors, this study is one of the few studies to use SEM for analysis of VCT and demonstrate how various factors interact to influence the intention and the final behaviour of coming for VCT.

\section{REFERENCES}

1. UNAIDS/WHO. AIDS UPDATE, December 2004. Geneva, Swizerland: WHO; 2004. Report No. 9291733903.

2. WHO/UNAIDS. UNAIDS Report 2005. Geneva: WHO; 2005.

3. World Health Organisation (WHO). Progress on global access to HIV antiretroviral therapy: an update on "3 by 5". Geneva: WHO; 2005 June.

4. Ehrnst A, Zetterstro R. Vertical transmission of HIV-1 infection and dilemma of infant feeding. Acta paediatr. 2003; 92:990-1002.

5. Wilfert C. Prevention of mother to child transmission of HIV: reflection on implementing of PMTCT in the developing world. Acta paediatr. 2002; 91:863-5.

6. Maman S, Mbwambo J, Hogan NM, Kilonzo GP, Sweat M. Women's barriers to HIV-1 testing and disclosure: challenges for HIV-1 voluntary counselling and testing. Aids Care. 2001 Oct; 13(5):595-603.

7. Coates TJ, Grinstead OA, Gregorich SE, Heilbron DC, et al. Efficacy of voluntary HIV1 counselling and testing in individuals and couples in Kenya, Tanzania, and Trinidad: a randomised trial. Lancet. 2000 JUL 8; 356(9224):103-12.

8. Creese A, Floyd K, Alban A, Guinness L. Costeffectiveness of HIV/AIDS interventions in Africa: A systematic review of the evidence. The Lancet. 2002 May 11; 359 (9318):1635.

9. Sweat M, Gregorich S, Sangiwa G, Furlonge C, et al. Cost-effectiveness of voluntary HIV-1 counselling and testing in reducing sexual transmission of HIV-1 in Kenya and Tanzania. Lancet. 2000 JUL 8; 356(9224):113-21.

10. Matovu JK, Gray RH, Makumbi F, Wawer M J, et al. Voluntary HIV counselling and testing 
acceptance, sexual risk behaviour and HIV incidence in Rakai, Uganda. AIDS. 2005; 19(5):503-11.

11. Ezumah NN. Gender issues in the prevention and control of STIs and HIV/AIDS: lessons from Awka and Agulu, Anambra State, Nigeria. Afr J Reprod Health. 2003 Aug; 7(2):89-99.

12. Fylkesnes.K, Siziya S. A randomized trial on acceptability of voluntary HIV counselling and testing. Trop Med Int Health. 2004 MAY; 9(5):566-72.

13. Jiraphongsa C, Danmoensawat W, Greenland $\mathrm{S}$, Frerichs R, et al. Acceptance of HIV testing and counselling among unmarried young adults in Northern Thailand. AIDS Educ Prev. 2002 Apr; 14(2):89-101.

14. Kawichai S, Celentano DD, Chaifongsri R, Nelson KE, Srithanaviboonchai K, Natpratan $\mathrm{C}$, et al. Profiles of HIV voluntary counselling and testing of clients at a district hospital, Chiang Mai Province, northern Thailand, from 1995 to 1999. J Acquir Immune Defic Syndr. 2002 Aug 15; 30(5):493-502.

15. Kawichai S, Nelson KE, Natpratan C, Celentano DD, et al. Personal history of voluntary HIV counselling and testing (VCT) among adults aged 19-35 years living in periurban communities, Chiang Mai, Northern Thailand. AIDS Behav. 2005 Jun; 9(2):233-42.

16. Kawichan C. HIV incidence and risk behaviours after voluntary HIV counselling and testing (VCT) among adults aged 19-35 years living in peri-urban communities around Chiang Mai city in Northern Thailand, 1999. Aids Care. 2000; 16(1):21-35.

17. Muller O. Sexual risk behaviour reduction associated with voluntary HIV counselling and testing in HIV infected patients in Thailand. Aids Care. 1995; 7(5):567-71.

18. Wolff B, Nyanzi B, Katongole G, Ssesanga D, Ruberantwari A, Whitworth J. Evaluation of a home -based Voluntary counselling and testing intervention in rural Uganda. Health Policy plan. 2005; 20(2):109-16.

19. Eichler MR, Ray SM, Del Rio C. The effectiveness of HIV post-test counselling in determining healthcare-seeking behaviour. Aids. 2002 Apr 12; 16(6):943-5.

20. Glick P. Scaling Up HIV Voluntary Counseling and Testing in Africa. What Can
Evaluation Studies Tell Us About Potential Prevention Impacts? Evaluation review. 2005; 29(4):331-57.

21. SU I Hou. Sexual behaviour and risk perception related to HIV infection among college students. /J.annepidem. 2004(10).

22. Kakoko DC, Astrom AN, Lugoe WL, Lie GT. Predicting intended use of voluntary HIV counselling and testing services among Tanzanian teachers using the theory of planned behaviour. Soc Sci Med. 2006 Aug;63(4):9919.

23. Berg MB, Mimiaga MJ, Safren SA. Mental health concerns of HIV-infected gay and bisexual men seeking mental health services: an observational study. Aids Patient Care 2004/11; 18(11):635-43.

24. Coleman C.L. The Contribution of Religious and Existential Well-Being to Depression among African American Heterosexuals with HIV Infection. Issues in Mental Health Nursing. 2004; 25(1):103-10.

25. Griffin KW, Rabkin J G, Remien R H, Williams JBW. Disease severity, Physical limitations and depression in HIV-infected men. Journal of Psychosomatic Research. 1998 1998/2; 44(2):219-27.

26. Judd F, Komiti A, Chua P, Mijch A, Hoy J, Grech P,et al. Nature of depression in patients with HIV/AIDS. Aust N Z J Psychiatry. 2005; 39:826-32.

27. WHO/UNAIDS. Policy statement on HIV testing. Geneva, Switzerland: UNAIDS; 2004.

28. Michelo C, Sandoy IF, Fylkesnes K. Marked HIV prevalence declines in higher educated young people: evidence from populationbased surveys (1995-2003) in Zambia. Aids. 2006 Apr 24; 20(7):1031-8.

29. Victims web. Beck Depression Inventory (BDI). 2006 [cited 2006 01/07] available from:http://www.swin.edu.au/victims/resourc es/assessment/affect/bdi.html

30. Byrne B, Barbara. Testing for Multigroup Invariance Using AMOS Graphics: A Road Less Travelled. Structural Equation Modelling. 2004; 11(2):272-300.

31. Acton SG. Great Ideas in Personality. 2006 [cited 2006 10/02]; Available from: h $\mathrm{t} \quad \mathrm{t} \quad \mathrm{p}: \mathrm{l} / \mathrm{w}$ w $\mathrm{w}$ personal.umich.edu/ ppa/mlaguide/urlabc/g.h $\underline{\text { tml }}$ 
32. Linn JG, Poku KA, Cain VA, Holzapfel KM, Crawford DF. Psychosocial outcomes of HIV illness in male and female African American (learned helpless) clients. Social Work Care. 1995; 21(3):43-60.

33. Boswell D, Baggaley R. Voluntary counselling and testing (VCT) and young people. Geneva: Family Health International; 2002.

34. Wilson TE, Jaccard J, Levinson RA, Minkoff $\mathrm{H}$, Endias R. Testing for HIV and Other Sexually Transmitted Diseases: Implications for Risk Behaviour in Women. Health Psychology. 1996 1996/7; 15(4):252-60.

35. De Cock KM, Marum E, Mbori-Ngacha D. A serostatus-based approach to HIV/AIDS prevention and care in Africa. The Lancet.2003 2003/11/29; 362(9398):1847-9.

36. Haidet P, Stone DA, Taylor WC, Makadon HJ. When risk is low: primary care physicians \& rsquo; counselling about HIV prevention. Patient Education and counselling. 2002 2002/1; 46(1):21-9.

37. Bollen K, Lennox R. Conventional wisdom on measurement: A structural equation perspective. Am J Psychol. 1991; 33(2909/91):305-12. 